\title{
VORTEX MODELS AND BOUNDARY LAYER INSTABILITY*
}

\author{
ALEXANDRE JOEL CHORIN†
}

\begin{abstract}
Random vortex methods are applied to the analysis of boundary layer instability in two and three space dimensions. A thorough discussion of boundary conditions is given. In two dimensions, the results are in good agreement with known facts. In three dimensions, a new version of the method is introduced, in which the computational elements are vortex segments. The numerical results afford new insight into the effects of the third dimension on the stability of a boundary layer over a flat plate.
\end{abstract}

Key words. vortex, boundary layers, random walk, three-dimensional instability

Introduction. The random vortex method as described in Chorin [7] is intended for the approximation of flows at high Reynolds number $R$. Its main features are as follows: (i) the nonlinear terms in the Navier-Stokes equation are taken into account by a detailed analysis of the inviscid interactions between vortices of small but finite core ("vortex blobs"), (ii) viscous diffusion is taken into account by adding to the motion of the vortices a small random Gaussian component of appropriate variance, and (iii) no-slip boundary conditions are approximated by a vorticity creation algorithm. Fuller details are given below. Developments, modifications, and applications of the method can be found e.g. in Ashurst [1], Chorin [10], [11], Leonard [26], [27], McCracken and Peskin [30], Shestakov [36]. Theoretical analysis can be found in Hald [18], Hald and Del Prete [19], and Chorin et al. [12].

This grid-free method is suitable for the analysis of flow at high Reynolds number because it has no obvious intrinsic source of diffusion. Most approximation methods solve equations which are close to the equations one wants to solve; the difference consists of higher order terms multiplied by small parameters. This is also the form of the diffusion term, and as a result, in most methods, the effects of a small $R^{-1}$ are dominated by numerical effects and the physics of high Reynolds number flow are suppressed. In vortex methods, the misrepresentation of the higher harmonics which occurs in the usual discretization methods (which usually has a diffusive effect among other effects) is replaced by the misrepresentation of the interaction of neighboring vortices (an essentially inviscid phenomenon which is a source of error, but not of diffusive error). In the absence of the nonlinear terms, the diffusion is approximated on the average exactly. Thus one may hope that the results of the calculation approximate the flow at whatever Reynolds number was intended, albeit with a statistical error, rather than at some other lower Reynolds number intrinsic to the algorithm. A good guess at the solution of the problem one wants to solve is better than an unbiguous solution of the wrong problem.

The method produces a flow field which is random. The error in the calculation is the sum of two parts: the expected value of the computed solution differs from the true solution, and any realization of the computed solution (or more accurately, any functional thereof) differs from the expected value by a random amount which can be estimated by its standard deviation (see e.g., Lamperti, [25]). The expressions for these quantities will be given below, when the appropriate notation will be available.

In the present paper we apply random vortex methods to the analysis of the boundary layer over a flat plate in two and three space dimensions. The calculations

* Received by the editors May 15, 1979. This work was supported in part by the Engineering, Mathematical, and Geosciences Division of the U.S. Department of Energy through the Lawrence Berkeley Laboratory.

† Department of Mathematics, University of California, Berkeley, California 94720. 
have two main objects. In the two dimensional case we shall show that the vortex method exhibits a physical instability at an appropriate Reynolds number. The ability to do so is of course a basic requirement for any method which claims to have some use at high Reynolds number. The specific problem we apply our method to has a simplifying feature, inasmuch as the location of the sharp gradients is known in advance to be near the wall, and thus the equations of motion can be solved in two dimensions by finite difference or other non-statistical methods in appropriately scaled variables. The interesting fact about our calculation is that it does not require such preliminary scaling of the variables, i.e., the random walk can be relied upon to create the appropriate diffusive length scale.

The second main goal of our calculation is to use the method to investigate the much harder problem of boundary layer instability in three dimensions, and in particular, two of the striking features of its solution: The formation of streamwise vortices and the creation of active spots. The three dimensional calculation requires a generalization of our method, and both the two dimensional and three dimensional problems afford the opportunity to use an improved algorithm for imposing the boundary conditions accurately.

In the next four sections we present the calculation in two dimensions. In later sections we present the three dimensional calculations.

The physical problem in two dimensions. Consider a semi-infinite flat plate placed on the positive half-axis, with an incompressible fluid of density 1 occupying the half space $y \geqq 0$. At time $t<0$ the fluid is at rest. At $t=0$, the fluid is impulsively set into motion with velocity $U_{\infty}$. The flow is described by the Navier-Stokes equations,

$$
\begin{aligned}
& \partial_{t} \xi+(\mathbf{u} \cdot \nabla) \xi=R^{-1} \Delta \xi, \\
& \Delta \psi=-\xi, \\
& u=\partial_{y} \psi, \quad v=-\partial_{x} \psi,
\end{aligned}
$$

where $\mathbf{u}=(u, v)$ is the velocity vector, $\mathbf{r}=(x, y)$ is the position vector, $\xi$ is the vorticity, $\psi$ is the stream function, $\Delta \equiv \nabla^{2}$ is the Laplace operator, and $R$ is the Reynolds number, $R=U_{\infty} L / \nu$, where $L$ is a length scale typical of the flow. The boundary conditions are

$$
\begin{aligned}
& \mathbf{u}=\left(U_{\infty}, 0\right) \text { at } y=\infty, t>0, \\
& u=v=0 \text { for } y=0, x>0, \\
& \frac{\partial v}{\partial y}=0 \text { for } y=0, x<0 .
\end{aligned}
$$

Initially, $\mathbf{u}=\left(U_{\infty}, 0\right)$ everywhere.

If $\boldsymbol{R}$ is large, the Prandtl boundary layer equations should provide a reasonable description of the flow near the plate and away from the leading edge. These equations can be written in the form [Schlichting [35], Chorin [10], [11]],

$$
\begin{aligned}
& \partial_{t} \xi+(\mathbf{u} \cdot \nabla) \xi=\nu \partial_{y}^{2} \xi, \\
& \xi=-\partial_{y} u, \\
& \partial_{x} u+\partial_{y} v=0 .
\end{aligned}
$$

where $\xi, u, v, x, y$ have the same meaning as in equations (1), and $\nu$ is the viscosity. If $U_{\infty}=1$ and $L=1, R=\nu^{-1}$. The boundary conditions for equations (2) are: $u=U_{\infty}$ for $y=\infty, \mathbf{u}=0$ for $y=0$. Equations (2) have a stationary solution, the Blasius solution, which is a function of the similarity variable $\mu=y / \sqrt{x \nu}$. Let the displacement thickness $\delta$ 
be defined by

$$
\delta=\int_{0}^{\infty}\left(1-u / U_{\infty}\right) d y
$$

the corresponding Reynolds number is $R_{\delta}=U_{\infty} \delta / \nu$. In Blasius flow, $\delta=1.72 \sqrt{\nu x}$, and $R_{\delta}=1.72 \sqrt{x / \nu}$, where it is assumed that $U_{\infty}=1 . \delta$ and $R_{\delta}$ are increasing functions of $x$. For $R_{\delta} \geqq R_{\delta c}$ the Blasius solution is unstable to infinitesimal perturbations which satisfy equations (1) (see Lin [29]); $R_{\delta c} \simeq 520$, (See Jordinson [21]). These unstable modes are the Tollmien-Schlichting waves. The vortex interpretation of the waves is as follows: The boundary layer is a region of distributed vorticity imbedded in a shear flow. Vorticity imbedded in a shear tends to become organized into coherent macroscopic structures ("negative temperature states", "local equilibria", see Onsager [32], Chorin [8]). This tendency is counteracted by the diffusive effects. The latter become weaker as $x$ increases, since the vorticity gradients decrease as the layer spreads. Far enough downstream (i.e., for $\boldsymbol{R}_{\delta}$ large enough), the tendency towards coherence can overcome the diffusive effects; the Tollmien-Schlichting waves can be viewed as a weak train of organized vortex structures.

The value of $R_{\delta c}$ given above has to be lowered if the unperturbed flow is treated as a nonparallel flow and if edge effects are taken into account (Townsend [37]). More importantly, the boundary layer is unstable to perturbations of a finite amplitude for values of $R_{\delta}$ smaller than $R_{\delta c}$ (for analysis of similar situations, see Eckhaus [14], Meksyn and Stuart [31]). A survey of finite amplitude stability theory for the flat plate problem is given by Roshotko [33]). The boundary layer becomes more unstable if the outside flow is turbulent or contains vortical structures (see Schlichting [35], Rogler and Reshotko [33]). Since our calculation will by its very nature contain finite amplitude perturbations, vortices, a substantial amount of noise, and edge effects, the appropriate value of $\boldsymbol{R}_{\delta}$ which separates stable from unstable regimes is unclear. Presumably, there exists a value $R_{\delta c}^{\prime}$ such that for $R_{\delta} \leqq R_{\delta c}^{\prime}$ all perturbations decay; the best guess of $R_{\delta c}^{\prime}$ we can obtain by looking at the references above is $R_{\delta c}^{\prime} \simeq 300$, with a substantial margin of error. Cebeci and Smith [4] suggest a value $R_{\delta c}^{\prime} \simeq 320$.

For $R_{\delta} \geqq R_{\delta c}$, the perturbations can grow, but I found little information as to what they do in two dimensions; presumably they grow and reach some finite amplitude equilibrium; this is the typical situation in other two-dimensional stability problems, for example in the thermal convection problem (see e.g. Chorin [6]). All experimental studies I know deal with the more important and more realistic three dimensional problem which will be discussed further below.

The numerical methods in two dimensions. Consider first the Navier-Stokes equations (1) in the whole plane. Assume that $\xi=\sum_{j} \xi_{j}$, where the $\xi_{i}$ are functions of small support ( $\xi_{i}$ is a "blob"). Let $\psi=\sum \psi_{j}$, where $\Delta \psi_{j}=-\xi_{j}$. (If we had $\xi_{j}=\kappa_{j} \delta\left(\mathbf{r}-\mathbf{r}_{j}\right)$, $\kappa_{j}=$ constant, we would have concluded that $\psi_{j}=-\left(\kappa_{j} / 2 \pi\right) \log \left|\mathbf{r}-\mathbf{r}_{j}\right|$.) For $\xi_{j}$ smooth but of small support, let $\kappa_{j} \equiv \int \xi_{j} d x d y$, and we must have

$$
\lim _{|\mathbf{r}| \rightarrow \infty} \frac{\psi_{j}}{(1 / 2 \pi) \log \left|\mathbf{r}-\mathbf{r}_{j}\right|}=-\kappa_{j}
$$

For $\left|\mathbf{r}-\mathbf{r}_{j}\right|$ small, $\psi_{j}$ differs from $\left(\kappa_{j} / 2 \pi\right) \log \left|\mathbf{r}-\mathbf{r}_{j}\right|$ (or else it would introduce undesirable unbounded velocities, see Chorin [7], Hald [18]). We set

$$
\psi_{j}(\mathbf{r})= \begin{cases}\frac{\kappa_{i}}{2 \pi} \log \left|\mathbf{r}-\mathbf{r}_{j}\right|, & |\mathbf{r}| \geqq \sigma, \\ \frac{\kappa_{j}}{2 \pi} \frac{|\mathbf{r}|}{\sigma}+\text { const., } & |\mathbf{r}|<\sigma .\end{cases}
$$


This is the form introduced in Chorin [7]; it differs from the forms described by Hald in [18] for reasons which will become apparent below. Clearly $\xi_{j}=-\Delta \psi_{j}$ is of small support. $\sigma$ is a cut-off which remains to be determined.

Equations (1) state that the vorticity moves with the velocity field which it induces, i.e., let $\mathbf{u}_{j}=\left(u_{j}, v_{j}\right)$ be the velocity field induced by the $j$ th blob, and let $\mathbf{r}_{i}=\left(x_{i}, y_{i}\right)$ be the center of the $i$ th blob, then

$$
\frac{d \mathbf{r}_{i}}{d t}=\sum_{j \neq i} \mathbf{u}_{j}, \quad\left(\mathbf{u}_{j} \text { evaluated at } r_{i}\right)
$$

This equation can be approximated by

$$
\mathbf{r}_{j}^{n+1}=\mathbf{r}_{i}^{n}+k \sum_{j \neq i} \mathbf{u}_{j}
$$

where $k$ is a time step and $\mathbf{r}_{i}^{n} \equiv \mathbf{r}_{i}(n k)$. Hald [19] has shown that a higher order method is indeed more accurate but we shall use (4) for the sake of simplicity.

The heat equation is well known to be solvable by a random walk algorithm (see Chorin [7]). As a result, equations (1) can be solved by moving the blobs according to the law

$$
\mathbf{r}_{i}^{n+1}=\mathbf{r}_{i}^{n}+k \sum_{j \neq i} \mathbf{u}_{j}+\boldsymbol{\eta}
$$

where $\boldsymbol{\eta}=\left(\eta_{1}, \eta_{2}\right), \eta_{1}, \eta_{2}$ independent Gaussian random variables with mean 0 and variance $2 k / R$.

Suppose we wish to solve equations (1) in a domain $D$ with boundary $\partial D$. The normal boundary condition $\mathbf{u} \cdot \mathbf{n}=0$ on $\partial D, \mathbf{n}$ normal to $\partial D$, can be readily taken into account by solving $\Delta \psi=-\xi$ subject to the appropriate boundary condition, with the help of potential theory. In the case of flow over a flat plate, the method of images will do the job. The no-slip boundary condition $\mathbf{u} \cdot \mathbf{s}=0, \mathbf{s}$ tangent to $\partial D$, can be imposed through the creation of the appropriate amount of vorticity: Let $u_{0}$ be the velocity component tangent to the wall created by the algorithm as described so far, and suppose $u_{0} \neq 0$. The no-slip condition and the viscosity will create a boundary layer in which the total vorticity per unit length is

$$
\int_{\text {wall }}^{\text {interior }} \xi d n=\int \frac{\partial u}{\partial n} d n=u_{0}
$$

In the algorithm presented in [7], we reproduced this effect numerically by creating a vortex sheet of strength $u_{0}$ at the wall, dividing its vorticity among blobs, and allowing these blobs to participate in the subsequent motion of the blobs according to the laws (5). If a blob is created at every piece of boundary of length $h$, its intensity is

$$
\kappa=u_{0} h .
$$

If a blob inside the fluid happens to cross the boundary, it is removed. It should be apparent that the amount of vorticity created at each time step depends on the cut-off $\sigma$. If $\sigma$ is small, the backwash of the vortex may be large, and a vortex whose center is near the boundary will create a vortex whose intensity will have an opposite sign, etc. If $\sigma$ is large, the backwash of a newly created vortex may not be sufficient to annihilate $u_{0}$, and more vortices will be created, all of the same sign. Presumably, on the average the total amount of vorticity is independent of $\sigma$. The algorithm in this form is not accurate (see Chorin et al. [12]). This lack of accuracy as well as the desire to reduce the amount of 
computational labor have led to the formulation of the vortex sheet algorithm with which one can solve the boundary layer equations (2) (Chorin [10]). The computational elements are segments of a vortex sheet. Let $u_{0}$ be the velocity component parallel to the wall. A segment $S$ of a vortex sheet is a segment of a straight line, of length $h$, parallel to the wall, such that $u$ above $S$ differs from $u$ below $S$ by an amount $\xi$; ("above" means "further from the wall"), $u_{\text {above }}-u_{\text {below }}=\xi$. $\xi$ is the intensity of the sheet.

Consider a collection of $N$ segments $S_{i}$, with intensities $\xi_{i}, i=1, \cdots, N$. Let the center of $S_{i}$ be $\mathbf{r}_{i}=\left(x_{i}, y_{i}\right)$. To describe their motion, one begins with equations (2b) and (2c). Equation (2b) can be integrated in the form

$$
u(x, y)=U_{\infty}-\int_{y}^{\infty} \xi(x, \alpha) d \alpha
$$

where $U_{\infty}$ is the velocity at infinity seen by the layer. Equation (2c) yields

$$
v(x, y)=-\partial_{x} \int_{0}^{y} u(x, \alpha) d \alpha
$$

Equations (7a) and (7b) allow one to determine $u, v$ if $\xi=\xi(x, y)$ is given. One can visualize each sheet as casting a shadow between itself and the wall. The darker the shadow, the smaller $u$ becomes. Whatever fluid enters a shadow region from the left and cannot leave on the right must leave upwards. From equations (7) one can derive the following expression for $\mathbf{u}_{i}=\left(u_{i}, v_{i}\right)$ at the center $\mathbf{r}_{i}$ of the $i$ th sheet

$$
u_{i}=U_{\infty}-\frac{1}{2} \xi_{i}-\sum_{j} \xi_{j} d_{j}
$$

where $d_{j}=1-\left|x_{i}-x_{j}\right| / h$ is a smoothing function, and the summation is over all $S_{j}$ for which $0 \leqq d_{j} \leqq 1$ and $y_{j} \geqq y_{i}$. This is of course a small subset of all the sheets; only the sheets which lie in a narrow vertical strip around $u_{i}$ affect $u_{i}$. Similarly,

$$
v_{i}=-\left(I_{+}-I_{-}\right) / h
$$

where

$$
\begin{aligned}
& I_{ \pm}=U_{\infty}-\sum_{ \pm} \xi_{j} d_{j}^{ \pm} y_{j}^{*}, \\
& d_{j}^{ \pm}=1-\left|x_{i} \pm h / 2-x_{j}\right| / h, \\
& y_{j}^{*}=\min \left(y_{i}, y_{j}\right) .
\end{aligned}
$$

The sum $\sum_{+}\left(\right.$resp. $\left.\sum_{-}\right)$is over all $S_{i}$ such that $d_{j}^{+} \leqq 1$ (resp. $\left.d_{j}^{-} \leqq 1\right)$. The motion of the sheets is then given by

$$
\begin{aligned}
& x_{i}^{n+1}=x_{i}^{n}+k u_{i}, \\
& y_{i}^{n+1}=y_{i}^{n}+k v_{i}+\eta .
\end{aligned}
$$

These formulas are analogous to (4); $\eta$ is a Gaussian random variable with mean 0 and variance $2 \nu k$; it appears only in the $y$ component because equations (2) take into account diffusion in the $y$ direction only.

This vortex sheet algorithm generates a velocity field $\mathbf{u}=(u, v)$ which satisfies the boundary condition $u=U_{\infty}$ at $y=\infty, v=0$ at $y=0$. The no-slip boundary condition $u=0$ at $y=0$ can be satisfied by the following vorticity generation procedure (see [10]): Continue the flow from $y>0$ to $y<0$ by antisymmetry, i.e., $\mathbf{u}(x,-y)=-\mathbf{u}(x, y)$. Since $\xi=-\partial u / \partial y$, and both $u$ and $y$ change signs, we have $\xi(x,-y)=\xi(x, y)$; if $u(x, 0)=u_{0} \neq$ 0 , we also have a vortex sheet of strength $2 u_{0}$ at the wall. This sheet can be divided into 
segments and allowed to participate in the subsequent motion. The antisymmetry can be imposed by reflecting any sheet which crosses the wall back into the fluid. One can require that all the sheets created satisfy the requirement $\left|\xi_{i}\right| \leqq \xi_{\max }$, where $\xi_{\max }$ is some reasonably small quantity. To do this, one may have to create more than one sheet at any one point at any given time step. The sheet method can be modified to make it more efficient and to reduce the variance of the results (see [10]). The interaction of the sheets is not singular and no cut-off is needed. The amount of vorticity created at the wall is unambiguous, and the cost of the calculation is small. This is of course balanced by the fact that the Prandtl equations are not uniformly valid approximations to the NavierStokes equations, and the transition from sheets to blobs involves in general a decision process which in turn is not unambiguous.

Note that the antisymmetry just described cannot be used directly with the vortex blob method. Indeed, if $\mathbf{u}(x,-y)=-\mathbf{u}(x, y)$, it does not follow in general that

$$
\xi(x,-y) \equiv\left(-\frac{\partial u}{\partial y}+\frac{\partial v}{\partial x}\right) \quad \text { at }(x,-y)=\xi(x, y),
$$

since $x$ does not change sign. Thus, to impose the boundary conditions accurately on the blob method we shall have to use the sheet method as a transition near the wall, see below.

The version of the sheet method that we shall use is almost identical to the one described in [10] and documented in detail in Cheer [5]; this includes tagging and variance reduction techniques. The only difference is the following: In the earlier program, sheets were created at the wall, and on the average, half of them disappeared at each step. In the present program, we make exactly half of them disappear at each step and this reduces the total number of sheets retained. This is accomplished as follows: At each point at which sheets are created, their intensity is adjusted so that their number is even. A rejection technique (Handscomb and Hammersley [20]) is then used to ensure that any two successive $\eta$ 's used at the wall will have differing signs. This rejection technique can be used only at the wall, or else it would destroy the independence of the successive $\eta$ 's in the interior and thus fail to describe the diffusion process correctly.

The sheets and the blobs are objects of a very similar nature; they are determined by the same parameters, position and intensity. A computational element $\left(x_{i}, y_{i}, \xi_{i}\right)$ can be treated as either a sheet or a blob, depending on the circumstances. A sheet of negative intensity casts a shadow which slows the fluid under it; by the equation of continuity, this creates an upward flow to the left and a downward flow to the right, just as if the sheet were a vortex. The circulation around a sheet of intensity $\xi$ is $\xi h$, and if the sheet becomes a blob, the latter's intensity must be $\kappa=\xi h$, in agreement with equation (5).

These facts can be used to create a transition between the blobs and the wall. Pick a length $l$ such that a blob has a small probability of jumping more than $2 l$ in one random jump, i.e. $l$ a multiple of the standard deviation $\sqrt{2 k / R}$ of $\eta$. Any vortex which finds itself less than $l$ from the boundary (inside or outside) becomes a sheet and is reflected accordingly, and also taken into account accordingly when $u_{0}$ is computed. If a blob is further outside the domain than $l$ it is removed (presumably this happens rarely). If a sheet is inside the domain and its distance from the boundary is more than $l$ it becomes a blob again.

The cut-off $\sigma$ remains to be determined. A natural condition to impose is the following: consider a collection of blobs. As they approach each other and the boundary, their interaction should converge to the interaction of the corresponding 
sheets. Consider a sheet of intensity $\xi$ at $(X, Y)$, as well as vortex of intensity $\xi h$ at $(X, Y)$, together with its image vortex at $(X,-Y)$ required to satisfy the boundary conditions (the sheets need no images). If $\sigma=h / \pi$, the velocity fields induced along the vertical line $x=X$ are identical (Fig. 1). The lateral effects will tend to each other as $y \rightarrow 0$. Thus, if $\sigma=h / \pi$, the interaction of the blobs will approximate the interaction of the sheets when the blobs approach the boundary. Hence $\sigma=h / \pi$ is a natural choice for $\sigma$. Note that the form (3) of $\psi$ ensures that for $|\mathbf{r}| \leqq \sigma$ the magnitude of $\mathbf{u}$ is constant. This is the reason (3) is used. Remarks: (i) the value of $\sigma$ is twice the value used in [7]. (ii) The choice of $\sigma$ has the greatest effect near the wall, and thus it is natural to determine the value of $\sigma$ by considering what happens near the wall. (iii) Our value of $\sigma$ is large compared to the mean distance between blobs which is of order $R^{-1 / 2}$; this is in agreement with the requirements in Hald's proof. In summary the computational elements should be viewed as sheets near the wall, and as blobs far from the wall.

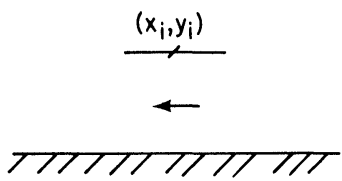

(a)

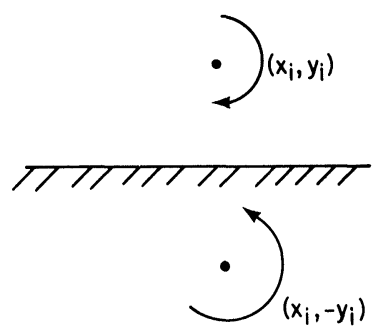

(b)

FIG. 1. Sheets and vortices near a wall.

A heuristic error analysis in [7] provides error estimates for the expected value of the velocity field produced by our methods in the form: error $=O(k)+O\left(R^{-1 / 2}\right)$, $R=$ Reynolds number based on a velocity and length scales typical of the flow away from the wall. Hald's analysis of the inviscid case suggests that this could be reduced to $O\left(k^{2}\right)+O\left(R^{-1 / 2}\right)$ if the time integration were carried out more accurately. The standard deviation of a smooth functional of the velocity should be $O\left(R^{-1 / 2}\right)$.

Application of the numerical method in two dimensions. In this section we describe the application of the vortex methods to the specific problem at hand. Note that if the sheet method is used by itself on the flat plate problem and if it converges in the mean to a stationary solution of the Prandtl equations (2); that solution is a function of the similarity variable $\mu$ only; more specifically, if two computer runs are made, with the same numerical parameters $k, h, \xi_{\text {max }}$, etc., the same sequence of random numbers, and the same impulsive initial conditions, but with two distinct values of $\nu$, the resulting computed solutions will be identical for equal values of $y / \sqrt{x \nu}$ and $x$. These facts are straightforward consequences of equations (8) (see Chorin and Marsden [11]). As a consequence, the instability of the boundary layer cannot be seen with the sheet method, and our main tool will be the blob method. We shall use the sheet method for the following limited purposes: (i) to provide a rational argument in favor of the value $\sigma=h / \pi$; (ii) as a vorticity creation algorithm, (iii) as a way of imposing an approximate Blasius flow before allowing unstable modes to grow; and (iv) as a diagnostic tool.

The number of vorticity elements required to describe the flow is large, since enough of them must be included to resolve the Tollmien-Schlichting waves, and those 
have a short wave length. From linear stability theory (see e.g. [29], [21]) one finds that the wave number of unstable Tollmien-Schlichting waves is between roughly $0.1 / \delta$ and $0.4 / \delta$ for moderate values of $R_{\delta}$, say very roughly $0.3 / \delta=0.2 / \sqrt{x \nu}$. The corresponding wave length is $\sim 10 \pi \sqrt{x \nu}$; the number of waves between 0 and $x$ is roughly $x$ divided by $10 \pi \sqrt{x \nu}$, i.e. $\sim R_{\delta} / 50$. The first unstable modes occur when $R_{\delta} \sim 500$, i.e., one has to be able to resolve at least 10 waves between the leading edge and the first occurrence of growing modes. One can also see that the time period is correspondingly small. For this reason stability calculations based on the Navier-Stokes equations are very expensive indeed (see e.g. Fasel [15]).

There is an additional constraint in the present work. It is interesting to compare the behavior of the growing modes in two dimension with the corresponding behavior in three dimensions; the two cases are quite different, and the contrast is very instructive when one is interested in the transition to turbulence. We wish to use comparable numerical parameters in two and in three dimensions, so that the comparison of the results be believable; the cost of three dimensional calculations is of course much larger even than the cost of two dimensional calculations; we must therefore look for ways of representing the boundary layer which are as economical as possible and yet exhibit a correct behavior.

There is no obvious way in which the steady Blasius profile can be imposed exactly on our array of vortex elements at the initial time. On the other hand, a calculation which starts from impulsive initial data contains a large and rather long-lived transient component whose behavior is not easily distinguishable from that of a growing mode. Part of this problem can be removed as follows: Start the calculation by using the sheet representation only (which is cheap and allows no instability), and run for a time $0<t<T_{0}, T_{0}$ large enough so that the Blasius profile will have been reached with some not unreasonable accuracy. At time $t=T_{0}$ allow some or all of the sheets to become blobs. In all the two dimensional runs described below we set $T_{0}=1$.

It is quite obvious that we shall not be able to duplicate the results of linearized stability theory. The initial data will not coincide exactly with the Blasius solution. The perturbations will not be small. In Fasel [15] the perturbation amplitude was about 0.05 of the free-stream velocity-an impossibly low level for our method. Our results should be compared with the behavior of finite amplitude perturbations in noisy flow. The advantages of our numerical method can be seen from the fact that the method requires no scaling. The very same program can be used to solve an interior flow problem. The algorithm provides its own scaling and concentrates the computing effort where it is needed. This should be particularly important in other problems where thin shear layers occur at locations which are not known in advance.

In the calculations described below, the vorticity is created at walls in the form of sheets, with all $\left|\xi_{i}\right| \leqq \xi_{\max }$. If the amount of vorticity needed to satisfy the boundary conditions is less than $\xi_{0}$, no sheets are created; here, $\xi_{0}=\xi_{\max } / 2$. When sheets find themselves at $y>l$ at time $t>T_{0}$, they become blobs; they become sheets again if $y<l$. $l$ must be such that the probability that $\eta>2 l$ is small. We checked that as long as $l \sim 1.5 \times$ the standard deviation of $\eta$, the results were insensitive to the value of $l$. Detailed calculations were performed for $0 \leqq x \leqq 1$; i.e., the typical streamwise length $L$ is 1 , and thus $R=U_{\infty} L / \nu=\nu^{-1}$. Both sheets and blobs were followed for $x>1$ but allowed to move only with the random component in their laws of motion. When they reached $x=X$ they were deleted. This was done to ensure that the right boundary at $x=1$, which is introduced only for computational convenience, behaves as an absorbing boundary and does not affect adversely the calculations in the region of interest $0 \leqq x \leqq 1$. We usually picked $X=2$. 
The interaction of two elements at least one of which was a sheet, was computed as if both were sheets. Two blobs interacted as blobs. In the computation of the tangential velocity at the wall, all elements were treated as sheets.

After much experimentation we picked $\xi_{\max }=0.6$. This is a large value of $\xi_{\max }$ and produces a crude and noisy boundary layer; however, it is sufficient for exhibiting the main effects. A relatively large value of $\xi_{\max }$ reduces the number of elements in the calculation, and, as explained above, this is of particular importance since we intend to present a three dimensional calculation. The choices of $h$ and $k$ are described in the next section.

In the steady state, the drag $D(x)$ on the piece of boundary between 0 and $x$ can be computed by the momentum defect formula (see e.g. [35]).

$$
D(x)=\int_{0}^{\infty} u\left(U_{\infty}-u\right) d y, \quad u=u(x, y) .
$$

The normalized drag is defined as

$$
d(x)=D(x) / D_{0}(x),
$$

where $D_{0}(x)$ is the Blasius drag $D_{0}(x)=0.6641 \sqrt{x \nu}$, which can be obtained from the Blasius solution. The velocities for use in formulas such as (10) are computed as if all the elements were sheets. We shall use $d(x)$ defined by (10) as a measure of the amplitude of the growing modes even when the flow is not steady and $D(x)$ is not really the drag on $[0, x]$.

Finally, we observed that if $k$ was too large the solution exhibited large oscillation of no possible physical significance. This is readily understood. We are solving a moderately large system of ordinary differential equations by Euler's method. The remedy is to reduce $k . k \leqq h$ is adequate.

Numerical results in two dimensions. In Table 1 and Fig. 2 we display the normalized "drag" $d(x)$ at $X=1 / 2$ as a function of $R$ and $t .(d(X)$ is the ratio $D(X) / D_{0}$, see formula (10) above). These calculations were made with $k=h=0.05$;

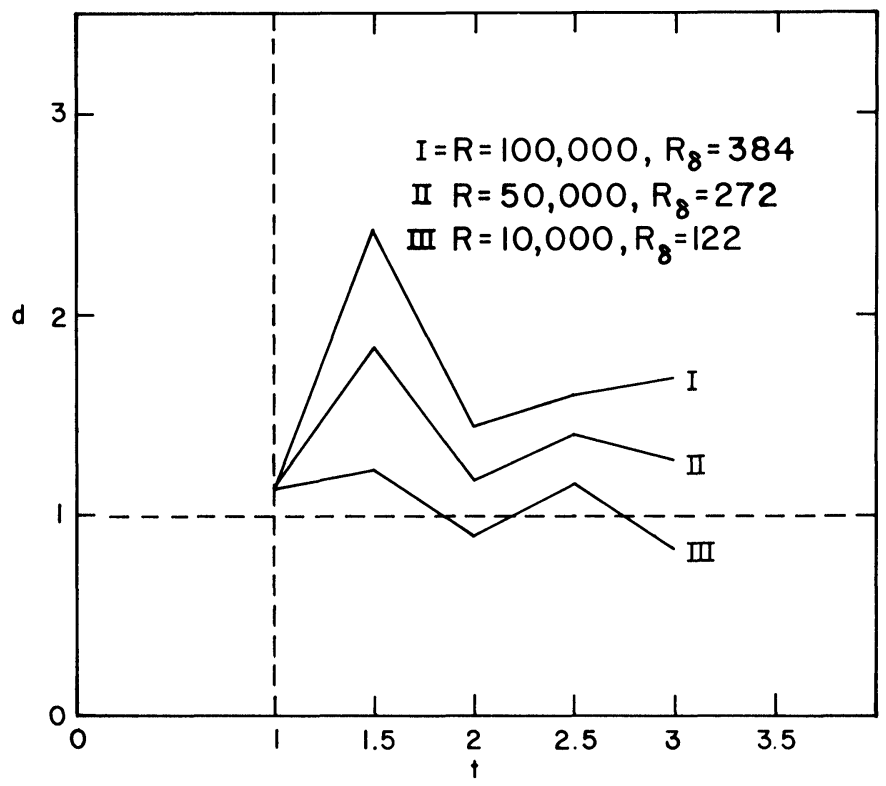

FiG. 2. Growth of an unstable layer in two dimension. 
TABLE 1

Drag as a function of Reynolds number and time.

\begin{tabular}{lccc}
\hline \multicolumn{1}{c}{$\boldsymbol{t}$} & $\begin{array}{l}R=10000 \\
(R \delta=122)\end{array}$ & $\begin{array}{c}R=50000 \\
(R \delta=272)\end{array}$ & $\begin{array}{c}R=100000 \\
(R \delta=384)\end{array}$ \\
\hline 1 & 1.11 & 1.11 & 1.11 \\
1.5 & 1.23 & 1.87 & 1.97 \\
2 & 0.89 & 1.18 & 1.39 \\
2.5 & 1.15 & 1.44 & 1.57 \\
3 & .77 & 1.25 & 1.65 \\
\hline
\end{tabular}

the other parameters are as described in the preceding section: $\xi_{\max }=0.6, X=2$, $\sigma=h / \pi$. The point $X=\frac{1}{2}$ is in the middle of the region of interest. In our units, $\nu=R^{-1}$, and $R_{\delta}=\delta / \nu=1.72 \sqrt{R / 2}$. From Table 1 and Fig. 2 one can see that $d(X)$ is growing for $R_{\delta}=394, R=10^{5} ; d(X)$ is not growing for $R_{\delta}=122, R=10^{4}$, and $d(X)$ is initially excited but ultimately slowly decaying for $R_{\delta}=272, R=5 \times 10^{4}$. This last fact is debatable; the value $R_{\delta}=272$ seems to be the approximate value of $R_{\delta c}^{\prime}$. These results are reasonable in view of what is known from the theory and from experiments.

In Fig. 4 we exhibit the edge of the boundary layer as a function of $x$ for $t=3$, $R=10^{4}$. The edge is defined as the smallest value of $y$ for which $u=U_{\infty}$. The edge is not at infinity because we have finite number of vortex elements and thus the tail of the probability distribution of the locations of the elements is not accurately approximated. The layer is stable at this value of $R$, yet the edge is ragged and the layer appears to be "intermittent" (for a definition of intermittency see e.g. Cebeci and Smith [4]). The "intermittency" is due to the presence of discrete vortices; this connection will be

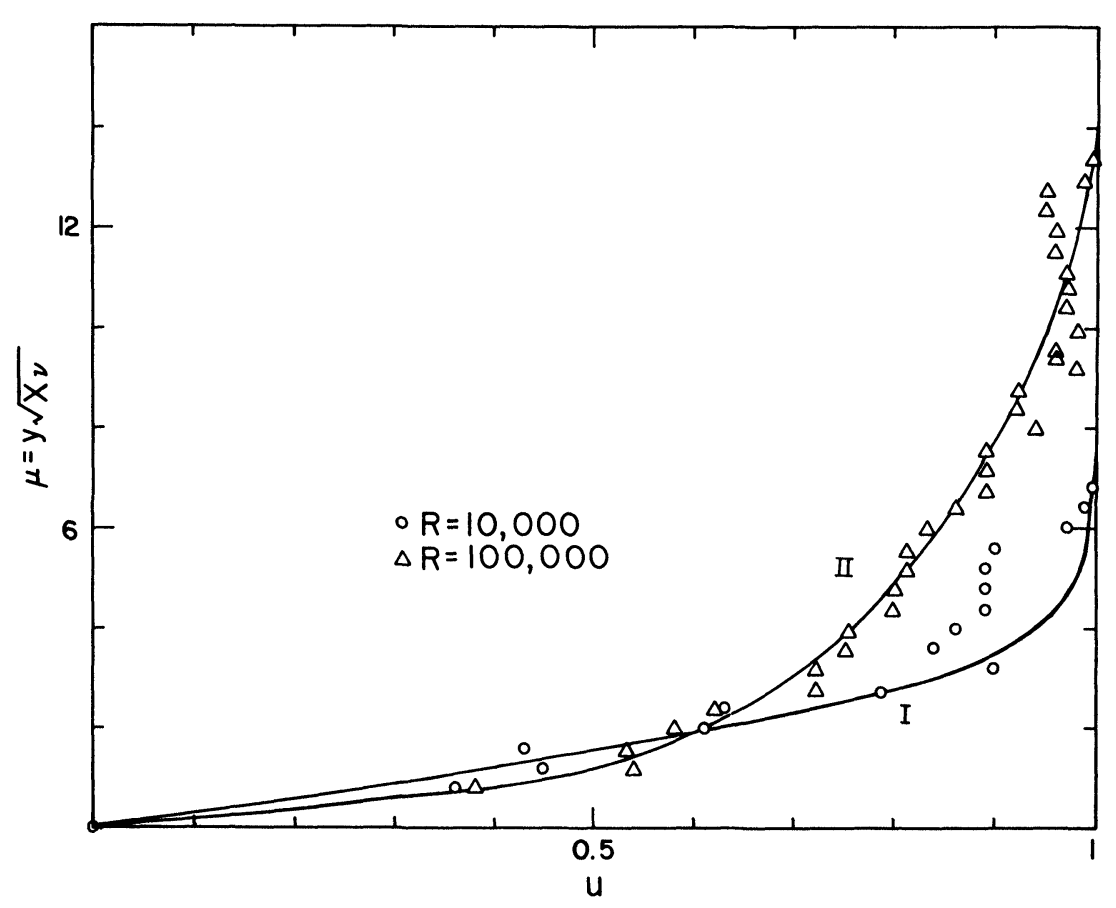

FIG. 3. Velocity profiles in two dimensions. 


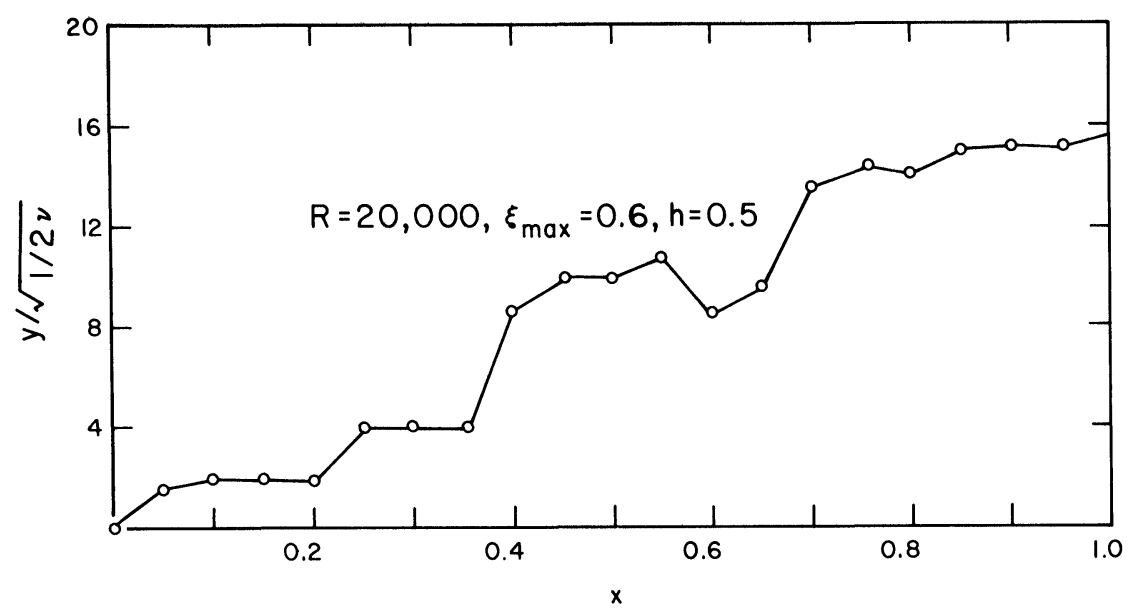

FIG. 4. Boundary layer shape.

exploited elsewhere for producing models of intermittency. It is obvious from Fig. 4 that the wave length of the growing modes cannot be determined directly from the instantaneous velocity distribution. However, it can be estimated indirectly. Consider the following question: how small must $h$ be to allow us to distinguish between stable and unstable layers? Suppose that for $h>h_{0}$ this distinction can be made, but for $h \leqq h_{0}$ the layer appears to be stable even when it should not be. Then $h_{0}$ is an estimate of the wave length of the growing modes, since when $h \leqq h_{0}$ these modes are suppressed. In Table 2 we present the values of $d(X)$ at $X=\frac{1}{2}$ as a function of $h$ for $R=10^{5}$. We see that $10<h_{0}<15$, in a reasonable if rough agreement with the Tollmien-Schlichting theory.

TABLE 2

Drag as a function of $h, R=100000, R_{\delta}=384$.

\begin{tabular}{|c|c|c|c|c|c|c|}
\hline \multirow{2}{*}{$h=1 / 20$, } & \multirow{2}{*}{$k=1 / 20$} & $t=1$ & 1.5 & 2 & 2.5 & 3 \\
\hline & & 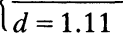 & 1.97 & 1.39 & 1.57 & \multirow[t]{3}{*}{1.65} \\
\hline \multirow{2}{*}{$h=1 / 15$, } & \multirow{2}{*}{$k=1 / 15$} & $t=1.27$ & 2 & 2.67 & 3.33 & \\
\hline & & $d=0.98$ & 1.48 & 1.66 & 1.70 & \\
\hline \multirow{2}{*}{$h=1 / 10$, } & \multirow{2}{*}{$k=1 / 10$} & $\{t=1$ & 2 & 3 & & \\
\hline & & $\mid d=0.98$ & 1.10 & 1.08 & & \\
\hline
\end{tabular}

In Fig. 3 we display the velocity as a function of $\mu=y / \sqrt{\nu X}$ at $X=\frac{1}{2}$ for $R=10^{4}$ and $R=10^{5}$, averaged over 10 steps between $t=2.5$ at $t=3$. Curve I is the laminar steady Blasius profile, and curve II was drawn in what appears to the eye as a reasonable neighborhood of the points obtained at $R=10^{5}$. The fluctuations are large (as one may well expect since $\xi_{\max }=0.6$ ), but the points at $R=10^{4}$ are in a reasonable agreement with the Blasius curve; curve II (an unstable case) has a different shape. The gradients are first sharper, then smaller than in the stable case. This is consistent with experience in the unstable regime of thermal convection (see e.g. [6]). It is also consistent with data for a turbulent boundary layer in the following sense: The Tollmien-Schlichting waves are large scale structures in comparison with boundary layer thickness, while in the 
stable regime there are no organized structures. In the turbulent regime one can associate a velocity with an eddy size; the changes in the profile due to the transition from the stable to the unstable regime should be of the same nature as the changes in the velocity profile which occur when the eddy size increases. This is indeed the case (see Favre et al., [16]; their data are reproduced in Lighthill, [28]).

A typical run from $t=0$ to $t=3$ with the numerical parameters used here took about 10 minutes on the UC Berkeley CDC 6400 computer. At the end of the calculation, there were about 200 sheets and 300 blobs.

The physical problem in three space dimensions. We now consider the three dimensional version of the preceding problem.

Consider a semi-infinite flat plate placed on the half plane $z=0, x>0$. A fluid of density 1 occupies the half space $z>0$. At time $t<0$ the fluid is at rest, at $t=0$ the fluid is impulsively set into motion with velocity $U_{\infty}=1$. The Navier-Stokes equations in three dimensional space can be written in the form:

$$
\begin{aligned}
& \partial_{t} \xi+(\mathbf{u} \cdot \nabla) \xi-(\xi \cdot \nabla) \mathbf{u}=R^{-1} \Delta \xi \\
& \xi=\operatorname{curl} \mathbf{u}, \\
& \operatorname{div} \mathbf{u}=0
\end{aligned}
$$

$\mathbf{u}=(u, v, w)$ is the velocity vector, and $\mathbf{r}=(x, y, z)$ is the position vector. The boundary conditions are

$$
\begin{aligned}
& \mathbf{u}=\left(U_{\infty}, 0,0\right) \text { for } z=\infty, t>0, \\
& \mathbf{u}=0 \text { for } z=0, x>0, \\
& \frac{\partial w}{\partial y}=0 \text { for } z=0, x<0 .
\end{aligned}
$$

Appropriate Prandtl equations can also be written. We shall need below only a simplified version of the equations, as well as the following fact about three dimensional boundary laminar layer approximations: The vertical component of the vorticity vanishes, i.e., for a solution of the Prandtl equations, $\boldsymbol{\xi}=\left(\xi_{1}, \xi_{2}, 0\right)$.

The Prandtl equations in three dimensions admit a two dimensional solution, the Blasius solution. That solution is unstable at high enough $R$. Squire's theorem (Lin, [29, p. 27]) states that the problem of instability to three dimensional infinitesimal perturbation is equivalent to a two dimensional problem at lower $R$.

Once the two dimensional perturbations begin to grow, several striking phenomena occur. In particular, before turbulence sets in, streamwise vortices (i.e. vortices whose axis is parallel to the mean flow) make their appearance. Intense secondary instabilities follow, and spots of intense motion emerge at random locations. An experimental investigation of boundary layer instability can be found in Klebanoff et al. [23]. Experimental investigations of turbulent boundary layers, in which phenomena resembling those which first arise immediately after the onset of instability persist and may be responsible for some of the observed features, are described e.g. in Favre et al. [16], Kline et al. [24], Willmarth [38]; theoretical aspects of several aspects of instability are found in Greenspan and Benney [17], Benney [3], Lighthill [28]. One of the major conclusions from the experimental data in Klebanoff et al., [23] is that the perturbed flow is periodic in the transverse direction (i.e., $y$ direction). It is therefore natural to consider in three dimensions equations (11) with the added periodicity conditions

$$
\mathbf{u}(x, y+q, z)=\mathbf{u}(x, y, z), \quad \xi(x, y+q, z)=\boldsymbol{\xi}(x, y, z),
$$


etc. Furthermore, from Klebanoff et al. (1962) we conclude that $q$ is roughly equal to the streamwise wave length of the first unstable Tollmien-Schlichting waves; roughly, $q=0.1$ in our units. We shall therefore be solving equations (11) with the boundary conditions (12) and (13), and $q=0.1$.

The numerical methods in three dimensions. We consider first the three dimensional analogue of the blob method. The three dimensional problem is more difficult because the vorticity $\boldsymbol{\xi}$ is now a stretchable vector quantity which must satisfy $\operatorname{div} \boldsymbol{\xi}=0$.

In earlier three dimensional calculations (Leonard, [26], [27], Del Prete [13], Chorin, (unpublished)), the vorticity field was represented as a sum of vortex filaments. The difficulties with this approach are: (i) a huge amount of bookkeeping is required to keep track of the changing vortex configurations; (ii) there is no obvious way to generate the filaments at the boundary in a consistent manner. We bypass these difficulties by representing the vorticity as a sum of vortex segments (Fig. 5). Each vortex segment moves in the flow field induced by all the others. The condition div $\xi=0$ will be satisfied only approximately. The segments have no independent physical significance. The two dimensional blobs do not have one either; physical vortices or vortex tubes are expected to emerge from the superposition of the computational blobs or segments. A segment $\Lambda$ is defined by seven quantities: The coordinates $\mathbf{r}^{(1)}=\left(x^{1)}, y^{(1)}, z^{(1)}\right)$ of the center of its base, the coordinates $\mathbf{r}^{(2)}=\left(x^{(2)}, y^{(2)}, z^{(2)}\right)$ of the center of its top, and its intensity $\kappa$. We shall write $\Lambda_{i}=\left(x_{i}^{(1)}, y_{i}^{(1)}, z_{i}^{(1)}, x_{i}^{(2)}, y_{i}^{(2)}, z_{i}^{(2)}, \kappa_{i}\right), i=1 \cdots, N, N=$ number of segments. The base and the top are circles of radius $\sigma$, (the cut-off), which will be determined below.

Given a vorticity yield $\boldsymbol{\xi}(\mathbf{r})$, the velocity field in a fluid which fills out the whole space is given by the Biot-Savart formula (see e.g. [2]):

$$
\begin{aligned}
& \mathbf{u}(\mathbf{r})=-\frac{1}{4 \pi} \int \frac{\mathbf{a} \times \boldsymbol{\xi}\left(\mathbf{r}^{\prime}\right)}{a^{3}} d \mathbf{r}^{\prime} \\
& \mathbf{a}=\mathbf{r}-\mathbf{r}^{\prime}, \quad a=|\mathbf{a}| .
\end{aligned}
$$

If the vorticity field is a sum of $N$ closed vortex lines with the $i$ th line having intensity $\kappa_{i},(14)$ becomes

$$
\mathbf{u}(\mathbf{r})=-\frac{1}{4 \pi} \sum_{i \neq 1}^{N} \kappa_{i} \int_{i \text { th line }} \frac{\mathbf{a} \times \mathbf{s}}{a^{3}} d s .
$$

$\mathbf{s}=\mathbf{s}\left(\mathbf{r}^{\prime}\right)$ is the unit tangent vector to the $i$ th line at $r^{\prime}, d s=d s\left(r^{\prime}\right)$ is the arc length along the $i$ th line, and as before $\mathbf{a}=\mathbf{r}-\mathbf{r}^{\prime}$. We now seek an interaction law between vortex segments which will approximate the motion induced by (14) or (15).

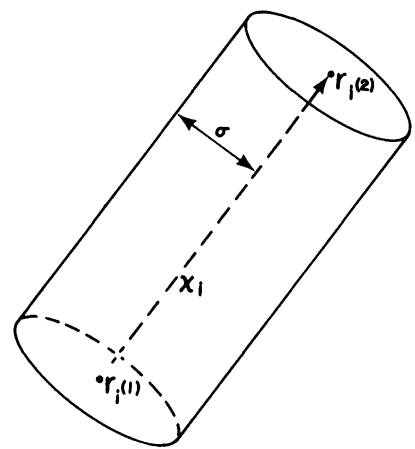

FIG. 5. A vortex segment. 
Inside the segment the velocity field must be kept bounded, just as is the case in two dimension. Furthermore, the field must be modified inside the segments in such a way that the segments will be compatible with the boundary calculations (see below). The problem of the finding the correct formulation of the vortex method in three dimensions is difficult, (see e.g. Leonard [27]). The formulation offered here is plausible but not rigorously justified.

We require that the motion of a vortex ring or line made up of vortex segments should preserve the shape of the ring or line. This can be accomplished by ensuring that the configuration of the vectors a and of the velocity vectors which enter the formula for the motion of the tips of the segment is the appropriate translate of the corresponding configurations which determine the motion of the bases. Thus, let $\Lambda_{i}, \Lambda_{j}$ be two vortex segments; define

$$
\begin{aligned}
& \mathbf{r}_{i}^{(1)}=\left(x_{i}^{(1)}, y_{i}^{(1)}, z_{i}^{(1)}\right), \quad \mathbf{r}_{i}^{(2)}=\left(x_{i}^{(2)}, y_{i}^{(2)}, z_{i}^{(2)}\right), \\
& \mathbf{s}_{j}=\mathbf{r}_{j}^{(2)}-\mathbf{r}_{j}^{(1)}, \\
& \mathbf{a}_{i j}^{(1)}=\mathbf{r}_{j}^{(1)}-\mathbf{r}_{i}^{(1)}, \mathbf{a}_{i j}^{(2)}=\mathbf{r}_{j}^{(2)}-\mathbf{r}_{i}^{(2)}, \quad \text { with } a_{i j}^{(1)}=\left|\mathbf{a}_{i j}^{(1)}\right|, \text { etc. }
\end{aligned}
$$

The velocity fields $\mathbf{G}_{i j}^{(1)}, \mathbf{G}_{i j}^{(2)}$ induced by $\Lambda_{j}$ at $\mathbf{r}^{(1)}$ and $\mathbf{r}^{(2)}$ will be approximated by:

$$
\text { If } \begin{aligned}
a_{i j}^{(1)} & \geqq \sigma \text { and } a_{i j}^{(2)} \geqq \sigma: \\
\mathbf{G}_{i j}^{(1)} & =\frac{-\kappa_{j}}{4 \pi} \frac{\mathbf{a}_{i j}^{(1)} \times \mathbf{s}_{j}}{\left(a_{i j}^{(1)}\right)^{3}}, \\
\mathbf{G}_{i j}^{(2)} & =\frac{-\kappa_{j}}{4 \pi} \frac{\mathbf{a}_{i j}^{(2)} \times \mathbf{s}_{j}}{\left(a_{i j}^{(2)}\right)^{3}}
\end{aligned}
$$

If either $a_{i j}^{(1)}<\sigma$ or $a_{i j}^{(1)}<\sigma$ :

$$
\begin{aligned}
\mathbf{G}_{i j}^{(1)} & =\frac{-\kappa_{j}}{4 \pi} \frac{\mathbf{a}_{i j}^{(1)} \times \mathbf{s}_{j}}{\sigma^{2} a_{i j}^{(1)}}, \\
\mathbf{G}_{i j}^{(2)} & =\frac{-\kappa_{j}}{4 \pi} \frac{\mathbf{a}_{i j}^{(2)} \times \mathbf{s}_{j}}{\sigma^{2} a_{i j}^{(2)}} .
\end{aligned}
$$

The equations of motion for each segment can now be obtained by summing the contributions of all the other segments and then adding to that sum the appropriate random component. This yields

$$
\begin{aligned}
& \mathbf{r}_{i}^{(1) n+1}=\mathbf{r}_{i}^{(1) n}+k \sum_{j \neq i} \mathbf{G}_{i j}^{(1)}+\boldsymbol{\eta}, \\
& \mathbf{r}_{i}^{(2) n+1}=\mathbf{r}_{i}^{(2) n}+k \sum_{j \neq 1} \mathbf{G}_{i j}^{(2)}+\boldsymbol{\eta},
\end{aligned}
$$

where $\mathbf{r}_{i}^{(1) n} \equiv \mathbf{r}_{i}^{(1)}(n k)$, etc., and $\boldsymbol{\eta}$ is a vector $\boldsymbol{\eta}=\left(\eta_{1}, \eta_{2}, \eta_{3}\right)$, with $\eta_{1}, \eta_{2}, \eta_{3}$, Gaussian random variable with means 0 and variances $2 k / R$, independent of each other. $\eta$ in (18a) is identical to $\eta$ in (18b), since diffusion does not introduce rotation or stretching. The boundary condition at the wall can be satisfied as before by the introduction of appropriate image segments.

One can write the boundary layer equation in three dimensions and solve them by a method in which the computational elements are pieces of a vortex sheet (= "tiles") with sides $h_{1}$ in the $x$ direction and $h_{2}$ in the $y$ direction. Each tile carries a two dimensional vortex with components $\xi_{1}, \xi_{2}$. As observed earlier, $\xi_{3}=0$ in the boundary layer equations. However, we shall use the tiles only near the boundary, where vortex 
stretching is presumably negligible, or to create an initial Blasius profile, in which stretching is exactly zero. Therefore, the boundary layer equations we shall be solving reduce to

$$
\begin{aligned}
& \partial_{t} \xi_{1}+(\mathbf{u} \cdot \nabla) \xi_{1}=\nu \frac{\partial^{2} \xi_{1}}{\partial z^{2}}, \quad \partial_{t} \xi_{2}+(\mathbf{u} \cdot \boldsymbol{\nabla}) \xi_{2}=\nu \frac{\partial^{2} z_{2}}{\partial z^{2}} \\
& \xi_{1}=\frac{\partial v}{\partial z}, \quad \xi_{2}=-\frac{\partial u}{\partial z}, \quad \operatorname{div} \mathbf{u}=0, \quad \mathbf{u}=(u, v, w) .
\end{aligned}
$$

These equations can be solved by a straightforward extension of the sheet method described earlier. No vortex stretching will be taken into account, and we shall not take the trouble to write out the equations in full. The rejection and variance reduction techniques carry over from the two-dimensional case. Care is taken to ensure that $\sqrt{\xi_{1}^{2}+\xi_{2}^{2}} \leqq \xi_{\max }$.

A tile created near the wall can become a segment if $t>T$ or if $z_{i}>l$. A segment which falls below $l$ becomes a tile again. The transformation of tiles into segments (and vice versa) must obey the following conditions:

(i) A tile must become a segment parallel to the wall; i.e., if a tile $\left(x_{i}, y_{i}, z_{i}, \xi_{1 i}, \xi_{2 i}\right)$ becomes a segment $\left(x_{i}^{(1)}, y_{i}^{(1)}, z_{i}^{(1)}, x_{i}^{(2)}, y_{i}^{(2)}, z_{i}^{(2)}, \kappa_{i}\right)$ we must have

$$
z_{i}^{(2)}-z_{i}^{(2)}=0 \text {. }
$$

(ii) A flow which is two dimensional when described by tiles must remain two dimensional when described by segments. The two dimensionality of a flow described by segments will be preserved only if the flow fields seen by the tips of the segments are translates of the flow fields seen by the bases, with a translation vector normal to the plane of the flow and pointing in the direction of a fixed normal $\mathbf{n}$ to that plane.

(iii) The stretching of the several segments represents the stretching of vorticity, which will be represented accurately only if the length of the segments is reasonably small. A reasonable normalization of that length in our problem is

$$
y_{i}^{(2)}-y_{i}^{(1)}=h_{2} \quad \text { when a segment is created. }
$$

(iv) The circulation around a vortex line made up of tiles must equal the circulation around a vortex line made up of segments. If $y_{i}^{(2)}-y_{i}^{(1)}$ is normalized by (19b) this requirement leads to

$$
\kappa_{i}=h_{1} \sqrt{\xi_{1 i}^{2}+\xi_{2 i}^{2}} \operatorname{sgn}\left(\boldsymbol{\xi}^{\prime} \cdot \mathbf{n}\right),
$$

where $\xi^{\prime}=\left(\xi_{1 i}, \xi_{2 i}\right)$, $\mathbf{n}$ is the fixed normal to the plane of the flow and $\operatorname{sgn}(\alpha)=1$ if $\alpha \geqq 0$, $\operatorname{sgn}(\alpha)=-1$ if $\alpha<0$.

The remaining connecting formulas between segments and tiles are obviously

$$
\begin{aligned}
& x_{i}^{(1)}=x_{i}, \\
& y_{i}^{(1)}=y_{i}, \\
& z_{i}^{(1)}=z_{i} .
\end{aligned}
$$

Formulas (19) are of course invertible, and the computational elements can be treated as either tiles or segments, as the occasion warrants.

When two segments interact, their interaction is given by formulas (19); when a segment and a tile interact, they are both viewed as tiles.

Finally, the cut-off must be determined. We must require that if we consider on one hand the interaction of two infinite vortex lines parallel to the $y$ axis represented by 
segments, and on the other hand the interaction of the same vortex lines represented by tiles, the former should approach the latter as the lines approach the wall. This requirement obviously reduces to the condition imposed on $\sigma$ in two dimensions, and yields $\sigma=h_{1} / \pi$. This conclusion is of course legitimate only if most of the vorticity does indeed point in a direction parallel to the $y$ axis.

Application of the numerical methods in three dimensions. In this section we discuss some of the features of the numerical method which are specific to the particular application at hand. Most of the numerical parameters are chosen just as they were chosen in the two-dimensional case; in particular, $l$ and $L$. We picked $h_{1}=k=\frac{1}{15}$, since the two-dimensional calculations showed that this was a minimal but adequate choice. We picked $h_{2}=q / 4$, after some experimentation showed that this value was sufficient to exhibit important effects.

The two major difficulties we encountered in three dimensions were: the large amount of computational labor, and the difficulty in imposing periodic boundary conditions on a grid-free method. The amount of labor is large not only because three-dimensional calculations are always more costly than two-dimensional calculations, but also (and especially) because the specific nature of the secondary instabilities which arise in three dimensions (see the next section) requires the creation of large amounts of vorticity at the walls. In consequence we used $\xi_{\max }=1$. This value seems to yield results which are compatible with two-dimensional results obtained with smaller values of $\xi_{\max }$, but it is obviously so large that one may legitimately argue that what we have is a model rather than an approximation.

Periodic boundary conditions can be imposed on a vortex calculation, but the price in computing labor is high. There again we did the least we could reasonably do. For each vortex segment with base located at $(x, y, z)$ (or its image created to satisfy the normal boundary condition, with a base at $(x, y,-z))$ we created two more segments, based at $(x, y \pm q, z), q=$ the period and took their velocity fields into account when we moved the segment. Similarly, new tiles must be created outside the strip $0 \leqq y \leqq q$ with locations and strengths determined by periodicity. Some rather complex programming is needed to keep track of the several image systems as the tiles become segments and vice versa.

Finally, we note that if $\xi_{1}=0$ at $t=0$, i.e., if there is no streamwise vorticity at all at $t=0$, none will ever be created by our algorithm. Thus, if we are to observe the effects of streamwise vorticity, we must introduce some by artificial means. We proceeded as follows: At $t=0$, for one time step, we changed the velocity at infinity. Instead of $\mathbf{u}(x, y, \infty)=\left(U_{\infty}, 0,0\right)$ we set

$$
\mathbf{u}(x, y, \infty)= \begin{cases}\left(U_{\infty}, A, 0\right) & \text { for } \frac{q}{4}<y<\frac{3 q}{4}, \\ \left(U_{\infty}, 0,0\right) & \text { elsewhere }\end{cases}
$$

We usually picked $A=10^{-3}$ (note that $U_{\infty}=1$ ). For $t>k$, we reverted to $\mathbf{u}(x, y, \infty)=$ $\left(U_{\infty}, 0,0\right)$ everywhere. The effect of this initial perturbation is to create a small streamwise vortex at the boundary, whose subsequent history is determined by diffusion, transport, and stretching.

Numerical results in three dimensions. Calculations done in three dimensions with $A=0$ (i.e. with no perturbation which could trigger three dimensional effects) produce results similar to the results of two dimensional calculations. They afford a check on both, but are not worth discussing separately. 


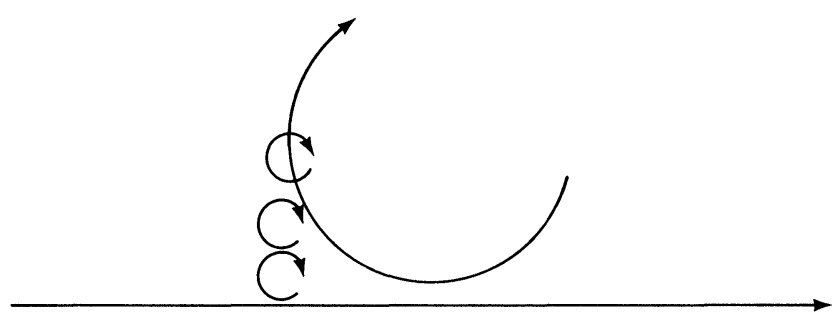

FIG. 6. Amplification of streamwise rotation.

Even a very small value of $\boldsymbol{A}$ (i.e. a very small three dimensional perturbation) has a substantial effect at all values of $R$ we tried. The first phenomenon one observes when $A \neq 0$ is that the boundary layer becomes thicker than in the case $A=0$. The mechanics of this effect are somewhat complex. A reasonable qualitative explanation is as follows: the rotation whose axis is parallel to the flow induces the creation of new streamwise vorticity at the wall. The new vorticity is then collected in streamwise strips in which the flow induced by the streamwise vortex leads away from the wall, while the regions where the induced flow points to the wall are depleted (see Fig. 6). The part of the boundary layer which thus expands can expand substantially, while the part which contracts cannot contract below zero. As a result the computed boundary layer thickness $\delta$ increases; $\delta$ at $(X, Y)$ is defined by

$$
\delta=\int_{0}^{\infty}\left(1-u(x, y, \alpha) / U_{\infty}\right) d \alpha
$$

In Figs. 7 and 8 we plot the ratio $\delta / \delta_{b}$ where $\delta=$ computed boundary layer thickness at $X=\frac{1}{2}$ averaged over a period in $y$, and $\delta_{b}=$ boundary layer thickness at $X=\frac{1}{2}$ computed from the steady Blasius solution. In Fig. 7, $R=20000$. Note that at $t=3, R_{\delta}$ computed with the steady $\delta$ is $R_{\delta}=187$, and thus the layer should be steady. However, if $R_{\delta}$ is evaluated with the computed boundary layer thickness, $R_{\delta}$ at $X=\frac{1}{2}$ is approximately 300 , and $R_{\delta}$ at $X=1$ is approximately 440 , well over the value at which the layer becomes unstable in the two-dimensional calculation. In Fig. $8, R=100000$,

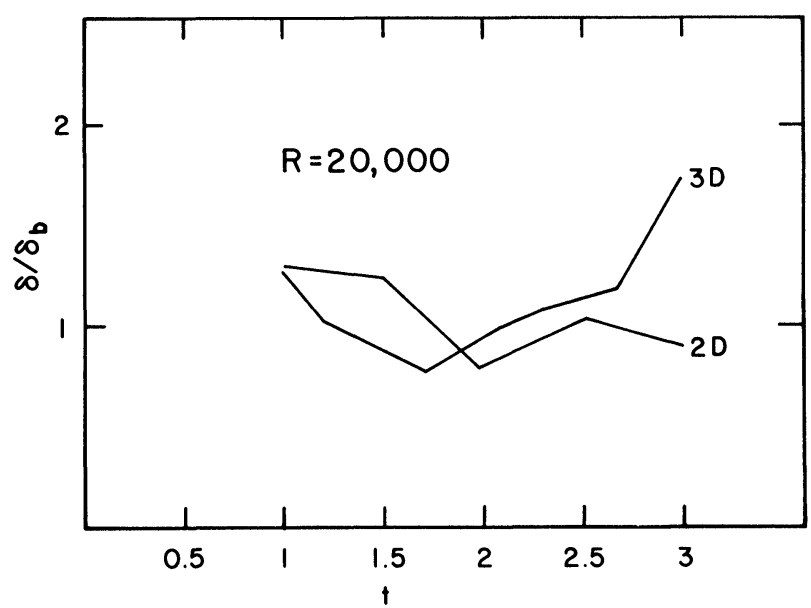

FIG. 7. Growth of boundary layer thickness, $R=20000$. 


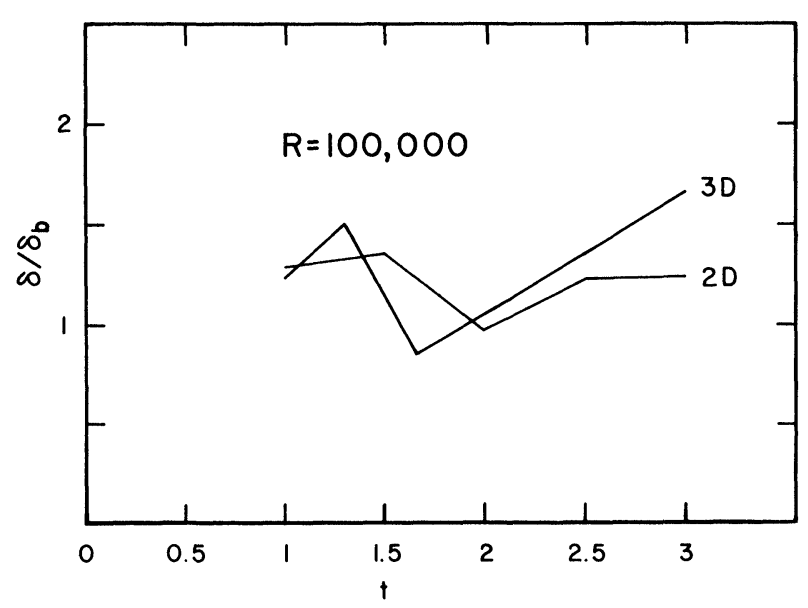

FIG. 8. Growth of boundary layer thickness, $R=100000$.

and the same effect is reproduced. The computed values of the drag are not greatly affected by this thickening of the layer. (This is quite plausible, in view of the extra factor $u$ in the integrand in the formula for the drag; the effect of this factor is to reduce the dependence of the drag on the velocity profile near the wall.)

When the layer becomes unstable to Tollmien-Schlichting waves, the streamwise vorticity begins to grow. The possible mechanisms for this growth are well known: The waves stretch lines; furthermore, they can create situations in which a horizontal streamwise line tilts away from the horizontal; its higher parts move faster than the lower parts, and stretching results. All segments are initially created with length $h_{2}$. If they stretch their length becomes $\left|\mathbf{r}_{i}^{(2)}-\mathbf{r}_{i}^{(1)}\right|$. The ratio $g=\left|\mathbf{r}_{i}^{(2)}-\mathbf{r}_{i}^{(1)}\right| / h_{2}$ is the stretching ratio. In Figs. 8 and 9 we plot $\bar{g}$, the average value of $g$, averaged over all segments. It is

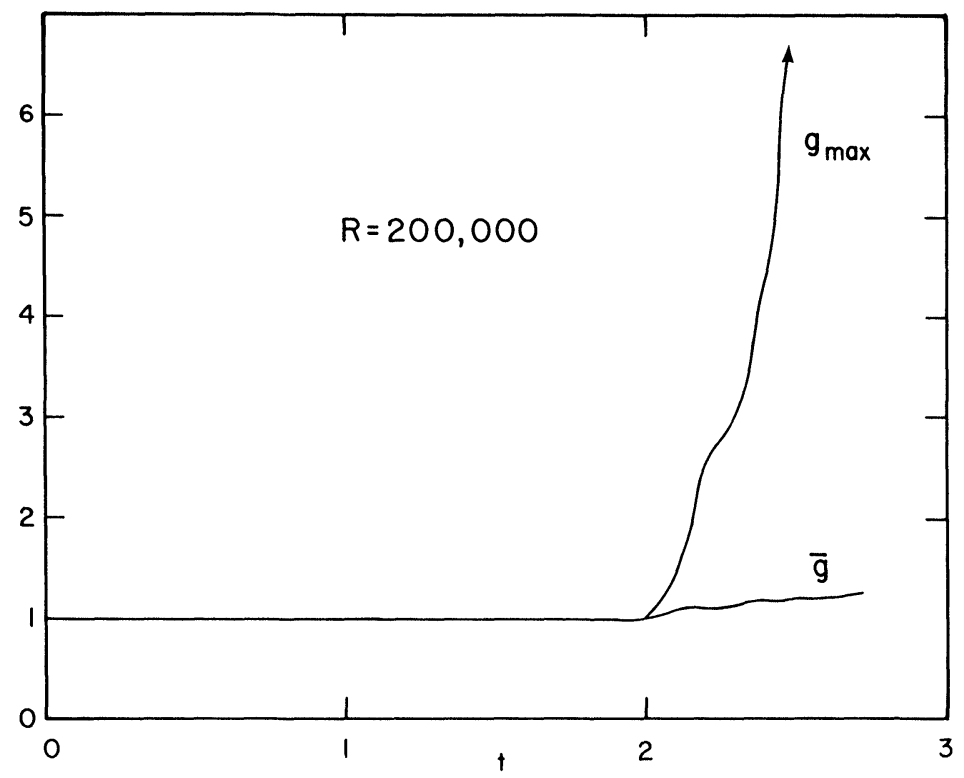

FIG. 9. Amplification of boundary layer disturbances in three dimensions, $R=20000$. 
seen to grow slowly with time. These figures are for $R=20000$ and 100000 . Note that at the time when $\bar{g}$ begins to grow with $R=20000$ the layer had become thicker as a result of secondary motion and $R_{\delta}$ is larger than the critical value. In Fig. 9 we also plotted $s$, the total streamwise vorticity, and $r$, the ratio of newly created streamwise vorticity to newly created transverse vorticity. Roughly, $r$ is an indication of the rate of growth of $s$. All these quantities are seen to grow slowly and steadily. The growth can be started earlier by increasing $A$. At value of $R$ smaller than 10000 , we never did succeed in inducing such growth within a time we could afford and without using very large values of $A$ (i.e. $A$ of order 1 -not a plausible value for our problem).

The more interesting graph in Figs. 9 and 10 is the graph of the maximum value $g_{\max }$ of the stretching ratio. This value can become very large $(\sim 17)$, i.e., some vortices are stretched by a large amount. This suggests an extraordinary spottiness of the stretching process. This spottiness can be explained as follows: because our method is random, the local velocity profile can differ from point to point. At some points the local profile may be much more unstable than at others, and as a result secondary instabilities, whose growth rate is very large (Greenspan [17]) will occur at some points and not at others. One can also argue that as a result of the variation in local profiles, at some points the segments may depart from the horizontal more than at others, and therefore the stretching mechanism is more intense there. These two explanations may of course be identical. The "spots" make the major contribution to the growth of the mean quantities. Their presence indicates that the layer contains a mechanism for amplifying greatly small differences in local conditions. However, one should remember that our numerical layer is much noisier than a real layer is likely to be.

In Table 3 we display the values of the streamwise component of $\mathbf{u}$ at $x=i h_{1}$, $y=j h_{2}, z=0$ and $R=2 \times 10^{5}, t=2.6$. The details of the fluctuations do not seem to have any particular physical significance. The values of $R$ and $t$ were picked somewhat arbitrarily; the table shows the spottiness of the field, and also shows that, as expected, the streamwise component of $\xi$ increases as the layer thickens.

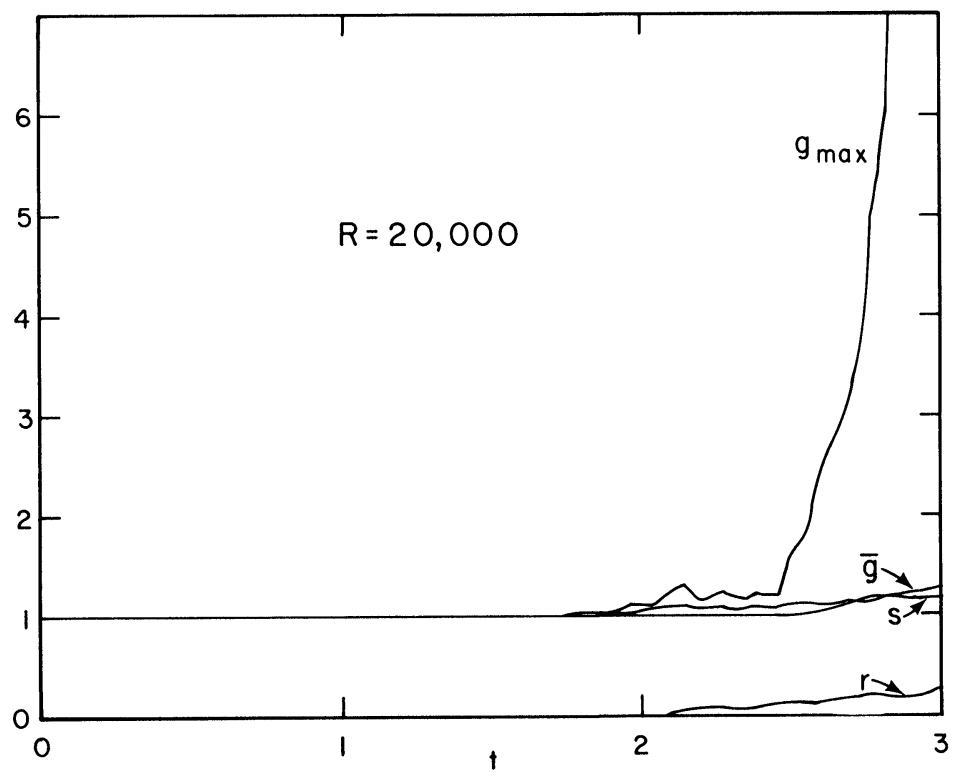

FIG. 10. Amplification of boundary layer disturbances in three dimensions, $R=100000$. 
When $g$ increases, more and more segments and tiles have to be created; this is why we needed a large value of $\xi_{\max }$. A further consequence is that computing for larger times than what we displayed is more expensive than we could afford. A typical run for $0 \leqq t \leqq 3$ took about one hour of CDC 6400 time at Berkeley.

TABLE 3

Streamwise vorticity at the boundary, $R=2 \times 10^{5}, t=2.6$

\begin{tabular}{rrrrr}
\hline & $j=1$ & 2 & 3 & 4 \\
$i=1$ & .000 & .000 & .000 & -.001 \\
2 & .000 & .000 & .000 & .000 \\
3 & .000 & .000 & .000 & .000 \\
4 & .003 & .000 & .000 & .000 \\
5 & .028 & .000 & .001 & .000 \\
6 & .000 & .000 & .000 & .001 \\
7 & -.046 & -.012 & .001 & .001 \\
8 & -.046 & -.004 & -.008 & -.010 \\
9 & -.038 & -.028 & -.002 & -.244 \\
10 & -.187 & -.054 & -.007 & -.093 \\
11 & .091 & -.065 & .030 & .076 \\
12 & -.062 & -.310 & .136 & .205 \\
13 & 2.260 & .507 & .480 & 1.070 \\
14 & -.077 & .150 & .826 & -.621 \\
15 & -.552 & .422 & .001 & -.273 \\
\hline
\end{tabular}

Conclusions. Our vortex methods, including the new three dimensional version and the new vorticity creation procedure, seem to be able to reproduce important features of boundary layer behavior in two and three dimensions and at Reynolds numbers where instability is expected. The three-dimensional calculation does exhibit a growth of streamwise vorticity as well as spottiness; however, it was not performed for times long enough for anything resembling fully developed turbulence to be present. Unlike other methods, our methods are not limited at high $R$ by the difficulty in distinguishing real from numerical diffusion; they are however limited, like other methods, by the fact that effects not resolved cannot be seen; i.e., if there are not enough computational elements to represent a phenomenon, that phenomenon will not be observed. Since fully turbulent flow is very complicated, our methods do not remove the need for careful modeling in some practical applications.

\section{REFERENCES}

[1] W. ASHURST, Numerical simulation of turbulent mixing layer dynamics, SANDIA (Livermore) Report (1977).

[2] G. K. BAtchelor, An Introduction to Fluid Mechanics, Cambridge University Press, London, 1967.

[3] D. J. BENNEY, A nonlinear theory for oscillations in a parallel flow, J. Fluid Mech., 10 (1960), p. 209.

[4] T. Cebeci AND A. M. O. Smith, Analysis of Turbulent Boundary Layers, Academic Press, New York, 1974.

[5] A. Cheer, Program BOUNDL, LBL-6443 Suppl. Report, Lawrence Berkeley Lab. (1978).

[6] A. J. CHORIN, A numerical method for solving incompressible flow problems, J. Comput. Physics, (1967), p. 12.

[7] -, Numerical study of slightly viscous flow, J. Fluid Mech., 57 (1973), p. 785.

[8] —, Gaussian fields and random flow, Ibid., 63 (1974), p. 21.

[9] - Lectures on Turbulence Theory, Publish/Perish, Boston (1976).

[10] —, Vortex sheet approximation of boundary layers, J. Comput. Physics, 27 (1978), 428. 
[11] A. J. CHORIN AND J. E. MARSDEN, A Mathematical Introduction to Fluid Mechanics, Springer Verlag, New York, 1979.

[12] A. J. Chorin, T. J. R. Hughes, M. F. MCCracken AND J. E. MARsden, Product formulas and numerical algorithms, Comm. Pure Appl. Math., 31 (1978), p. 205.

[13] V. DEL PRETE, Numerical simulation of vortex breakdown, LBL Math. \& Comp. Report 1978.

[14] W. EckHAus, Studies in Nonlinear Stability Theory, Springer Verlag, New York, 1965.

[15] H. FASEL, Investigation of the stability of boundary layers in a finite difference model of the Navier-Stokes equations, J. Fluid Mech., 78 (1970), p. 355.

[16] A. Favre, J. Gariglio AND J. Dumas, Phys. Fluids, 12, Suppl. (1967), p. 138.

[17] H. P. GREENSPAN AND D. J. BENNEY, On shear layer instability, breakdown and transition, J. Fluid Mech., 15 (1963), p. 133.

[18] O. HALD, The convergence of vortex methods II, SIAM J. Numer. Anal., 16 (1979), p. 726.

[19] O. HALD AND V. DEL PRETE, The convergence of vortex methods, Math. Comp., 32 (1978), p. 791.

[20] J. M. Hammersley AND D. C. Handscomb, Monte Carlo Methods, Methuen, 1964.

[21] R. JORDINSON, The flat boundary layer, Part I. Numerical integration of the Orr-Sommerfeld equation, J. Fluid Mech., 43 (1970), p. 801.

[22] H. T. KIM, S. J. KLINE AND W. C. REYNOLDS, The production of turbulence near a smooth wall in a turbulent boundary layer, Ibid., 50 (1971), p. 133.

[23] P. S. KLEBANOFF, K. D. TIDSTROM AND L. D. SARGENT, The three dimensional nature of boundary layer instability, Ibid., 12 (1962), p. 1.

[24] S. J. KLINE, W. C. REYNOLDS, F. A. SCHRAub AND P. W. RundstADLER, The structure of turbulent boundary layers, Ibid., 30 (1967), p. 741.

[25] J. LAMPERTI, Probability Theory, Benjamin, New York, 1966.

[26] A. LEONARD, Numerical simulation of interacting, three dimensional vortex filaments, Proc. 4th Int. Conf. Num. Methods Fluid Dynamics, Springer Verlag, New York, 1975.

[27] - Simulation of unsteady three dimensional separated flows with interacting vortex filaments, Proc. 5th Int. Conf. Num. Mech. Fluid Dynamics, Springer Verlag, New York, 1977.

[28] M. J. LighthILL, Turbulence, Osborne Reynolds and Engineering Science Today, Manchester Univ. Press, 1976.

[29] C. C. LIN, The Theory of Hydrodynamic Stability, Cambridge University Press, London, 1966.

[30] M. F. MCCRACKEN AND C. S. PESKIN, The vortex method applied to blood flow through heart valves, Proc. 6th Int. Conf. Num. Methods Fluid Dynamics, Springer Verlag, New York, 1978.

[31] D. Meksyn AND J. T. StuART, Nonlinear instability, Proc. Roy. Soc. London, A, 208 (1951), p. 517.

[32] L. ONSAGER, Statistical hydrodynamics, Nuovo Cimento, 6, Suppl. (1949), p. 229.

[33] E. ReSHOTKO, Boundary layer stability and transition, Ann. Rev. Fluid Mech., 8 (1976), p. 311.

[34] H. L. ROGLER AND E. RESHOTKO, Disturbances in a boundary layer introduced by a low intensity array of vortices, SIAM J. Appl. Math., 28 (1975), p. 431.

[35] H. SCHLICHTING, Boundary Layer Theory, McGraw-Hill, New York, 1960.

[36] A. I. Shest AKov, A hybrid vortex-ADI solution for flows of low viscosity, J. Comput. Phys., 31 (1979), p. 313.

[37] A. A. TownSEND, Boundary Layer Research, Freiburg Symposium, H. Gortler, Ed., Springer Verlag, 1958.

[38] W. W. WillmaRTH, Structure of turbulence in boundary layers, Advances in Appl. Mech., 15 (1976), p. 159. 